\title{
Direct Imaging of Exoplanets beyond the Radial Velocity Limit: Application to the HD 134987 System
}

\author{
Zhexing Li ${ }^{1}(1)$, Sergi R. Hildebrandt ${ }^{2,3}$ (1), Stephen R. Kane ${ }^{1}$ (1), Neil T. Zimmerman ${ }^{4}$ (10), Julien H. Girard ${ }^{5}$ (D), \\ Junellie Gonzalez-Quiles ${ }^{6}$ (D), and Margaret C. Turnbull ${ }^{7}$ (i) \\ ${ }^{1}$ Department of Earth and Planetary Sciences, University of California, Riverside, CA 92521, USA; zli245@ucr.edu \\ 2 Jet Propulsion Laboratory, California Institute of Technology, Pasadena, CA 91109, USA \\ ${ }^{3}$ Division of Physics, Mathematics and Astronomy, California Institute of Technology, Pasadena, CA 91125, USA \\ ${ }^{4}$ Exoplanets and Stellar Astrophysics Laboratory, NASA Goddard Space Flight Center, Greenbelt, MD 20771, USA \\ ${ }^{5}$ Space Telescope Science Institute, Baltimore, MD 21218, USA \\ ${ }^{6}$ Department of Earth and Planetary Sciences, Johns Hopkins University, Baltimore, MD 21218, USA \\ ${ }^{7}$ SETI Institute, Mountain View, CA 94043, USA \\ Received 2020 December 11; revised 2021 April 12; accepted 2021 April 13; published 2021 June 9
}

\begin{abstract}
Future direct imaging missions will primarily observe planets that have been previously detected, mostly via the radial velocity (RV) technique, to characterize planetary atmospheres. In the meantime, direct imaging may discover new planets within existing planetary systems that have bright enough reflected flux but insufficient signals for other methods to detect. Here we investigate the parameter space within which planets are unlikely to be detected by RV in the near future due to precision limitations but could be discovered through reflected light with future direct imaging missions. We use the HD 134987 system as a working example, combine RV and direct imaging detection limit curves in the same parameter space through various assumptions, and insert a fictitious planet into the system while ensuring that it lies between the RV and imaging detection limits. Planet validity tested through dynamical simulations and retrieval tests revealed that the planet could indeed be detected by imaging while remaining hidden from RV surveys. Direct imaging retrieval was carried out using starshade simulations for two mission concepts: the Starshade Rendezvous Probe, which could be coupled with the Nancy Grace Roman Space Telescope, and the Habitable Exoplanet Observatory. This method is applicable to all other systems and high-contrast direct imaging instruments and could help inform future imaging observations and data analysis on the discovery of new exoplanets.
\end{abstract}

Unified Astronomy Thesaurus concepts: Exoplanets (498); Direct imaging (387); Radial velocity (1332); Exoplanet systems (484); Exoplanet detection methods (489)

\section{Introduction}

The radial velocity (RV) technique is one of the most important exoplanet detection methods that led to the first exoplanet discoveries (Latham et al. 1989; Mayor \& Queloz 1995; Butler \& Marcy 1996). Besides making contributions to exoplanet detections, in recent decades, RV has also served as an important tool to characterize the Keplerian orbital elements, as well as to determine the masses of planets discovered by transit techniques, such as those by the Kepler (Borucki et al. 2010) and Transiting Exoplanet Survey Satellite (Ricker et al. 2015) missions. Discoveries by the Kepler mission (Borucki et al. 2011a, 2011b; Batalha et al. 2013; Burke et al. 2014; Mullally et al. 2015; Rowe et al. 2015; Coughlin et al. 2016; Thompson et al. 2018), as well as exoplanet occurrence rate studies (Howard et al. 2012; Petigura et al. 2013), indicate an increase in population for terrestrial planets, including those within the habitable zone (HZ; Kane et al. 2016). These planets require mass measurements from $\mathrm{RV}$ when transit timing variations are not available for proper characterization of physical properties and interpretation of atmospheric data (Batalha et al. 2019). Although some shortperiod low-mass planets could be recovered by RV with careful treatment, those with longer orbital periods would induce RV variations well below the sensitivities of current common spectrographs of a few meters per second (Fischer et al. 2016). With the advent of newly designed spectrographs such as NEID (Schwab et al. 2016) and ESPRESSO (Pepe et al. 2021) pushing the RV detection limit down to the tens of centimeters per second regime, low-mass terrestrial planet detections around nearby bright stars become even more tangible thanks to these extreme-precision RV instruments. However, without proper treatment of intrinsic stellar activity, noise such as stellar rotation, magnetic cycle, and jitter due to chromospheric activity may still present a challenge to the detection of lowmass planetary signals around the detection limits (Fischer et al. 2016; Luhn et al. 2020; Meunier \& Lagrange 2019, 2020). In addition, planets such as those that are further out from the host stars and Earth-sized terrestrial counterparts that lie in the HZ around solar-like stars could still remain below the detection threshold of RV in the near future.

Within the last decade, direct imaging has been evolving at a lightning pace in instrumentation and imaging processing techniques. This method's ability to directly image exoplanets with light reflecting straight from the planet's surface or atmosphere not only makes direct detection of the planet possible but also allows direct atmospheric retrieval and characterization (Feng et al. 2018; Damiano et al. 2020). High-contrast direct imaging from both the ground and space has successfully discovered several long orbital period giant planets (Chauvin et al. 2004; Marois et al. 2008; Lagrange et al.

2009), as well as measured emission spectra of several exoplanets (Janson et al. 2010; Konopacky et al. 2013). However, the current applications of direct imaging are limited to young, self-luminous giant planets that emit strong infrared 
emission or super-Jupiters that have a large angular separation from their host stars and are much inflated in their sizes.

These detections typically have contrast ratios around $10^{-4}$ to $10^{-5}$, while a Jupiter and an Earth analog would have contrast ratios on the order of $10^{-9}$ and $10^{-10}$, respectively. Future space missions such as the Nancy Grace Roman Space Telescope (hereafter Roman, formerly known as the Wide Field Infrared Survey Telescope; Spergel et al. 2015), a $2.4 \mathrm{~m}$ telescope, would be able to reach a predicted contrast ratio of a few times $10^{-9}$ to image and characterize the atmospheres of giant planets, thanks to the state-of-the-art starlight suppression enabled by a low-order wavefront sensing and control system for the internal coronagraph instrument (CGI; Trauger et al. 2016). If paired with the Starshade Rendezvous Probe (SRP), the instrument contrast could reach the required $1 \times 10^{-10}$ performance, or possibly even around $4.0 \times 10^{-11}$ (Seager et al. 2019; Romero-Wolf et al. 2021), making direct imaging of terrestrial exoplanets possible. More precisely, the instrument contrast of a starshade-telescope instrument at some angular distance from the starshade's center is defined as the amount of light at that angular distance averaged over a resolution element (e.g., within a point-spread function (PSF) FWHM) divided by the peak brightness of the light source as measured by that telescope when there is no starshade in place; see, e.g., Harness et al. (2021). A common choice for the angular distance used to report the starshade instrument contrast is the starshade (geometric) inner working angle (IWA), which is the angular radius of the starshade as seen from the telescope -72 mas in the blue band direct imaging channel of the SRP that we will consider in this work. This is the angular distance that corresponds to the $1 \times 10^{-10}$ and $4.0 \times 10^{-11}$ values just mentioned. The Habitable Exoplanet Observatory (HabEx; Gaudi et al. 2020) direct imaging concept, on the other hand, would be a $4 \mathrm{~m}$ class space telescope with an external starshade. Although the designed contrast ratio limit at the IWA of 70 mas would be similar to that of SRP, the larger primary mirror aperture, larger optical throughput, broader bandwidth, and greater resolving power could make detecting fainter and smaller terrestrial planets more feasible with HabEx.

Previous studies, including the design reports for both missions, have discussed the occurrence rate of different types of exoplanets and the predicted yield of detecting these planets throughout the missions. For the SRP with Roman, around one to two for both Earth- and Neptune-type planets and around two to three for Jupiter-like planets are expected to be detected (Seager et al. 2019; Romero-Wolf et al. 2021). The low predicted yield is due to the low optical throughput of the instrument, together with thrust and pointing constraints due to solar avoidance angles, that result in a small number of nearby stars, 20, to be surveyed by the mission (Feng et al. 2018; Seager et al. 2019; Romero-Wolf et al. 2021). The predicted yield for HabEx was much higher. In total, 55 rocky planets, 60 sub-Neptunes, and 63 giant planets are expected to be discovered by the HabEx mission (Gaudi et al. 2020). ${ }^{8}$

The expected mission yields shed light on possible direct imaging discoveries in systems that have known exoplanets previously discovered by other methods. Planets in the target list for characterization by future imaging missions will be

\footnotetext{
8 For details on the assumptions made and how predicted yields were calculated for both mission concepts, please refer to Seager et al. (2019) and Romero-Wolf et al. (2021) for SRP and Gaudi et al. (2020) for HabEx.
}

mostly RV planets, since they have been extensively observed by ground-based RV facilities and their Keplerian orbital parameters are well known. However, smaller RV signals caused by potential smaller planets in the system, if there are any, could be hidden below the detection threshold of current RV instruments. In this paper, we used the HD 134987 system, one of the more distant future imaging mission target systems $(26.2 \mathrm{pc})$, as a test case to determine if future direct imaging missions could detect low-mass planets that are hidden below the RV spectrograph sensitivities, as well as to test and compare the imaging capabilities of both Roman with SRP and HabEx on detecting such low-mass planets at this distance. We did this by injecting a fictitious planet into the system, for which the parameters are determined by combining both RV and direct imaging detection thresholds, carrying out dynamical simulations to determine the likely parameter space within which the fictitious planet could reside, and performing retrieval tests with realistic starshade imaging simulations to confirm that it is indeed below the RV detection threshold while it is still detectable by imaging. The use of both RV and direct imaging detection sensitivities in combination with dynamical analysis allows a full exploration of the parameter space within the HD 134987 system for the search for additional potential low-mass planetary objects. Although this paper focuses on the HD 134987 system only, similar work could be done for other systems, and the results could provide valuable information for future imaging missions regarding the chance of detecting an additional new planetary companion within known planetary systems.

In Section 2, we describe how the detection limits for both $\mathrm{RV}$ and direct imaging were calculated and the assumptions considered. In Section 3, we describe the dynamical simulation performed and the parameters selected for the fictitious planet. In Section 4, we present the derived expected planet-star flux ratios based on orbital parameters, realistic starshade images, and retrieval tests that include both $\mathrm{RV}$ and direct imaging. We discuss the results in Section 5 and conclude in Section 6.

\section{Detection Limits}

A better sense of the relationship between the detection thresholds of RV and direct imaging needs to be established before locating the possible regions where additional undetected planets could reside in a system. With increasing synergy between RV and direct imaging, having one detection limit plot that encompasses the detection threshold curves of both methods would be extremely useful and intuitive for realizing the detection sensitivity of known planets and predicting new discoveries. The RV detection threshold curves showing the different semiamplitude sensitivities of the spectrographs are typically displayed in the planetary mass versus orbital period parameter space, while for direct imaging, the detection limit curves for different imaging instruments are commonly shown in the planet-star contrast ratio versus angular separation/semimajor axis parameter space. To better visualize and directly compare the detection limits of the two, we made a few assumptions and put both detection limits in planetary radius versus separation parameter space.

\subsection{Radial Velocity}

For the RV detection limit, we used the instrumental precision of Keck HIRES (Vogt et al. 1994) and WYIN NEID 


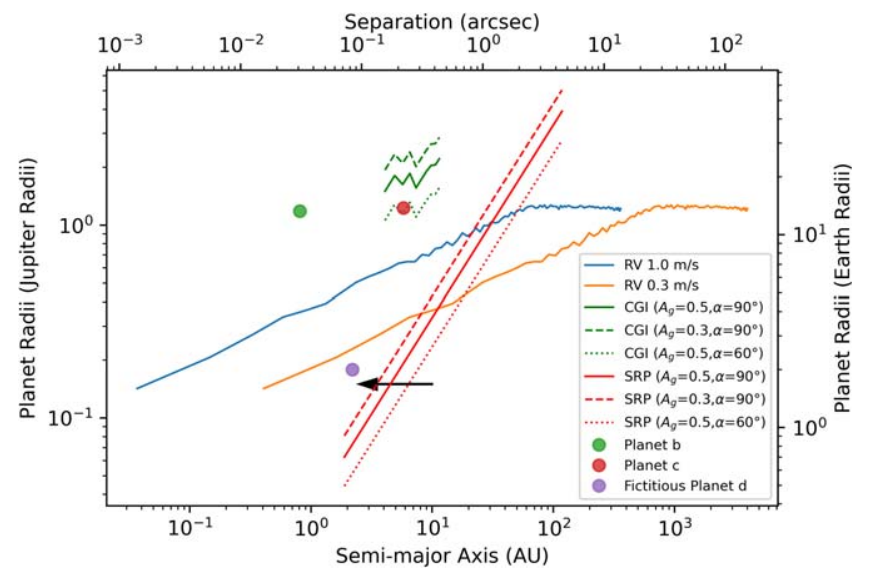

Figure 1. The RV and direct imaging detection thresholds of HD 134987 for different instruments in planetary radii and separation space. The RV limits are for 1 and $0.3 \mathrm{~m} \mathrm{~s}^{-1}$ precisions. Direct imaging thresholds are for Roman's CGI, as well as SRP. Also shown are the locations of two known planets in the HD 134987 system, along with the injected fictitious planet.

(Schwab et al. 2016) as the two RV standards, where each has a semiamplitude limit of about 1 and $0.3 \mathrm{~m} \mathrm{~s}^{-1}$, respectively. The RV semiamplitude can be expressed as

$$
K=\left(\frac{2 \pi G}{P}\right)^{1 / 3} \frac{M_{p} \sin i}{M_{t}^{2 / 3}} \frac{1}{\sqrt{1-e^{2}}},
$$

where $P$ is the period of the planet; $i$ is the inclination of the orbital plane relative to our line of sight, where an edge-on orbital plane is equivalent to $i=90^{\circ} ; e$ is the eccentricity of the planetary orbit; and $M_{t}=M_{*}+M_{p}$ is the combined mass of the star and the planet.

We constructed the RV detection limit for our test system HD 134987, starting in the planetary mass versus orbital period space. Rearranging Equation (1) for orbital period, we calculated the range of orbital period given $M_{p}$ 's range from 0.01 to $1 M_{\mathrm{J}}$ with a step of $0.01 M_{\mathrm{J}}$. Eccentricity is taken to be zero, and $M_{*}=1.10 M_{\odot}$, HD 134987's host star mass (Stassun et al. 2017). Varying eccentricity values show insignificant changes in the shapes or positions of the RV detection limit curves. Since the inclination for HD 134987 is unknown, we assumed a $45^{\circ}$ orbital inclination. The orbital period range obtained here can be converted to the corresponding semimajor axis range with ease. With the distance to the system of 26.20 pc (Gaia Collaboration et al. 2018), angular separation values were also computed. For the range of planetary mass values, we used FORECASTER (Chen \& Kipping 2017), a forecasting model that is based on a probabilistic mass-radius relation, to convert masses to planetary radii. The result of RV detection limits of 1 and $0.3 \mathrm{~m} \mathrm{~s}^{-1}$ in planetary radii and separation space can be seen in Figure 1. Although the RV curves extend into the extremely large separation $(>100 \mathrm{au})$, detection in this regime is extremely challenging, and the full range of $\mathrm{RV}$ curves derived from the input mass range was shown here only for easy comparison between the two sensitivity limits, as well as against the imaging limits described in Section 2.2. Highermass planets would have to orbit further away from the star in order to maintain the same semiamplitude level ( 1 and $0.3 \mathrm{~m} \mathrm{~s}^{-1}$ in this case), and the RV sensitivity curves flatten out at longer separations. This is due to planets in the Jovian regime with a dominating $\mathrm{H}-\mathrm{He}$ composition of the envelope that is massive enough where gravitational self-compression kicks in to stop the radius from increasing further (Guillot 2005). This is also reflected in Figure 3 of Chen \& Kipping (2017).

\subsection{Direct Imaging}

We used publicly available performance limits for both Roman's CGI and SRP as the direct imaging limits. The CGI detection limit curve is based on the instrumental direct imaging predicted performance at $575 \mathrm{~nm}$ with a total integration time of $100 \mathrm{hr}^{9}$ For SRP, we used the predicted performance values given in the science report (Seager et al. 2019; Romero-Wolf et al. 2021). Both contrast ratio limits are in flux ratio versus angular separation space. The conversion to estimated planetary radii versus separation space employs a Lambert sphere assumption for a planet, which assumes isotropic atmospheric scattering over $2 \pi \mathrm{sr}$. We used Equation (2) to estimate the planetary radii $R_{p}$ values based on the provided flux ratio $\epsilon(\alpha, \lambda)$ values, with the assumptions that the geometric albedo $A_{g}(\lambda)$ takes the values of 0.5 and 0.3 , representative of those of Jupiter and Neptune/terrestrial planet analogs in the visible band (Cahoy et al. 2010). The phase function $g(\alpha, \lambda)$, defined in Equation (3), was assumed to have a value equivalent to when a planet is at quadrature (phase angle $\alpha=90^{\circ}$ ) with respect to the star, at which the planet's location is feasible for carrying out direct imaging observations. This assumption is the same as that in the Roman CGI performance predictions. Thresholds for the $\alpha=60^{\circ}$ and albedo of 0.5 case are also shown for both CGI and SRP to demonstrate the effect of phase angle on planet detectability.

$$
\begin{gathered}
\epsilon(\alpha, \lambda) \equiv \frac{f_{p}(\alpha, \lambda)}{f_{*}(\lambda)}=A_{g}(\lambda) g(\alpha, \lambda) \frac{R_{p}^{2}}{r^{2}}, \\
g(\alpha, \lambda)=\frac{\sin \alpha+(\pi-\alpha) \cos \alpha}{\pi} .
\end{gathered}
$$

The combined detection limit curves of both RV and direct imaging in planetary radii and separation parameter space can be seen in Figure 1. Planets are detectable if they sit above the detection limit curve of a particular method or instrument; otherwise, the retrieval of the planetary signal would be challenging, as noise is likely to dominate. The locations of the two known giant planets in the HD 134987 system reveal valuable information; planet $\mathrm{c}$, one of the prime direct imaging targets for future space missions, sits within the field of view (FOV) of both CGI and SRP, while planet b orbits well outside the FOV of both and cannot be detected with these future missions, even though it reflects bright enough starlight. It is critical to point out from Figure 1 that CGI may not be able to detect planet $\mathrm{c}$ when the planet is at a phase angle of $90^{\circ}$ due to a lower planet-to-star flux ratio at this position. Only at smaller angular separation $\left(<60^{\circ}\right.$ phase angle, for example) will planet c become detectable for CGI. However, SRP will be able to see the planet throughout its entire orbit. This new way of displaying detection thresholds of RV and direct imaging for one system allows a direct and easy visual comparison of the sensitivities of instruments of different methods and the detectabilities of planets to each. It also gives useful insights into the possible locations where additional planets could be discovered by one method but not the other. We are interested

\footnotetext{
9 Data files regarding the performances of different direct imaging instruments, including Roman's, are created and hosted by Vanessa Bailey under Github repository https://github.com/nasavbailey/DI-flux-ratio-plot.
} 
in finding out if an additional planet may exist in our test system HD 134987 that is below the RV detection threshold but above the imaging contrast limit. This parameter space of interest in Figure 1 lies in the region below the RV $0.3 \mathrm{~m} \mathrm{~s}^{-1}$ curve and above the starshade limit (indicated by the arrow), in between the two existing giant planets (hereafter the Region). Although Figure 1 is informative in indicating the presence of such space, the curves were derived from the instrumental limits under the most ideal situations. For a more careful analysis of the allowed parameter space in which additional planets may reside in the Region, further simulations are needed that take into account dynamical interactions between planets, stellar jitter, spectrograph measurement error, imperfect starshade, local and exozodiacal light, and detector noise properties. These additional simulations are presented below.

\section{System Stability}

\subsection{Dynamical Simulation}

To explore the dynamical viability of the fictitious planet's orbit in the Region, we used the REBOUND (Rein \& Liu 2012) package to conduct dynamical simulations to search for locations where this fictitious planet may exist. We tested the viable orbits of this planet in between the orbits of the existing two planets using the Mean Exponential Growth of Nearby Orbits (MEGNO) indicator within REBOUND with the symplectic integrator WHFast (Rein \& Tamayo 2015). The MEGNO was originally developed by Cincotta \& Simó (2000) and can be applied to dynamical analysis from galactic scale to planetary systems. For our purpose, the MEGNO chaos indicator is useful in distinguishing the quasiperiodic or chaotic orbital time evolution of planetary systems of interest (Hinse et al. 2010). Because we are interested in locating the dynamically stable regions in between the discovered planets, the final MEGNO values returned for a grid of provided parameter space after numerically evaluating the MEGNO indicator with a given integration time would be useful in determining possible locations where the fictitious planet could dynamically exist. A high MEGNO value indicates the chaotic nature of the system, and it is unlikely for the orbits to remain dynamically stable over the long term.

We selected a radius value of $2 R_{\oplus}$ from Figure 1 for the fictitious planet that fits into the described region, and we use the mass-radius relationship tool FORECASTER (Chen \& Kipping 2017) to forecast an approximate mass of $5 M_{\oplus}$ for this planet, making it a sub-Neptune type of planet. The argument of periastron $(\omega)$ for this planet was set to $90^{\circ}$, and its orbit was assumed to be coplanar with the two known planets. We injected the fictitious planet into the system and ran the MEGNO simulation to test the stochasticity of the system at each grid point of the parameter space we provided. The grid, which can be seen in Figure 2, was set up with the eccentricity of the additional planet ranging from zero to 0.4 and semimajor axis values in between the two known planets, from 0.82 to $5.91 \mathrm{au}$. The orbital parameters of the two known planets were taken from the published values in Jones et al. (2010). A summary of the orbital parameters used for the two known planets is in Table 1. We ran the simulation with an integration time of 10 million $\mathrm{yr}$ and a time step of $0.03 \mathrm{yr}$ (10.95 days), consistent with the recommendation that the time step should be $1 / 20$ of the shortest orbital period in the system (Duncan et al. 1998). The integration was set to stop and return a large

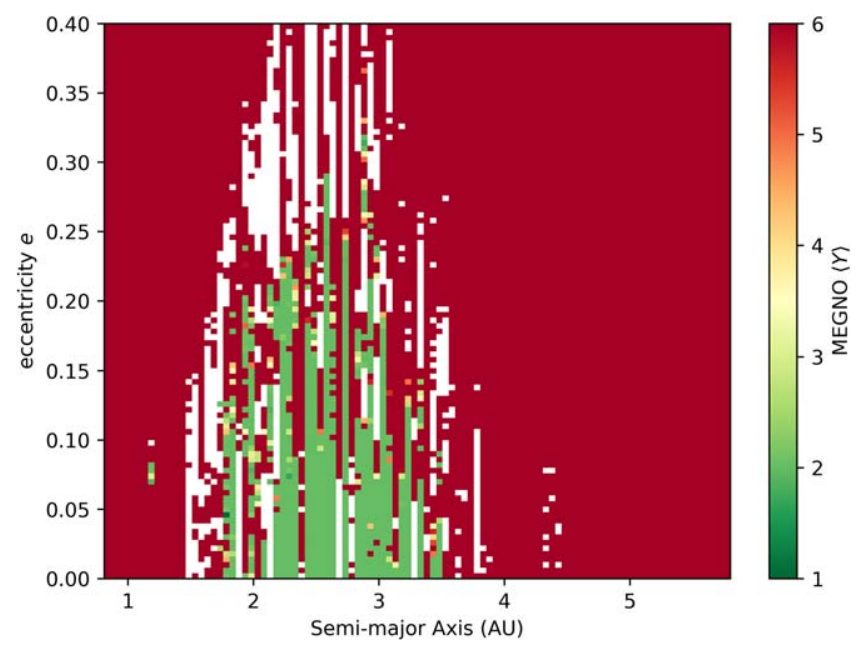

Figure 2. MEGNO simulation result of the HD 134987 system over 10 million yr with the inclusion of an additional planet in between the existing two. Horizontal and vertical ranges represent the semimajor axis and eccentricity values of the fictitious planet that MEGNO tested. Grids in green indicate a nonchaotic result. Red grids mark the locations of high chaos, while white ones represent close encounters or collisions; both are unfavorable locations for planetary long-term stability.

MEGNO value if the planet was ejected beyond $100 \mathrm{au}$. The result of the simulation returns final MEGNO values for each specific combination of semimajor axis and eccentricity, with a MEGNO value around 2 indicating a nonchaotic nature of the system and quasiperiodic motion of the planet if the planet was put at that location (Hinse et al. 2010).

The result of the MEGNO simulation is shown in Figure 2. The horizontal and vertical axes are the ranges of space the MEGNO simulations have tested. Grid points color-coded in green represent MEGNO values around 2, red indicates simulations that returned chaotic results, and other events, such as closer encounters and collisions that ended up with NaN simulation results, are in white. The MEGNO result indicates that a $5 M_{\oplus}$ fictitious planet that we injected into the system is unlikely to render the system chaotic in the long term, and the planet could have a stable orbit in between the existing two giant planets. The green grid points show that the stable orbit of the injected planet could be between $\sim 1.9$ and $\sim 3.5$ au with eccentricities between zero and $\sim 0.3$.

\subsection{Fictitious Planet Location}

We selected the semimajor axis and eccentric values for the fictitious planet according to the result of the MEGNO simulation and the location of the Region in Figure 1. The dynamically favored semimajor axis range of $1.9-3.5 \mathrm{au}$ according to the MEGNO simulation result sits comfortably within the Region in Figure 1, where it is below the RV thresholds but above those of SRP. Locations in Figure 1 near the detection limit curves would be subject to the noise contamination resulting in a much longer integration time, since they are near the instrumental limits. To avoid noise issues and ensure that our injected planet can be detected by imaging as easily as possible, we selected the semimajor axis value for our planet to be 2.2 au so that it resides well clear of the detection thresholds of SRP. At this distance, eccentricity could take any value up to around 0.2 , as Figure 2 indicated. For simplicity, we assigned a near circular eccentricity of 0.01 for the fictitious planet and kept other parameters unchanged. A 
Table 1

Orbital Parameters of Known and Injected Fictitious Planets in HD 134987

\begin{tabular}{|c|c|c|c|c|c|c|c|c|}
\hline Planet & $a(\mathrm{au})$ & $P$ (days) & $e$ & $\omega(\operatorname{deg})$ & $T_{p}(\mathrm{JD})$ & $\operatorname{Mass}\left(M_{\mathrm{J}}\right)$ & Radius $\left(R_{\mathrm{J}}\right)$ & Albedo \\
\hline $\mathrm{b}$ & 0.82 & 258 & 0.23 & 173 & $2,460,133$ & 2.25 & 1.19 & 0.5 \\
\hline $\mathrm{c}$ & 5.91 & 5000 & 0.12 & 15 & $2,461,100$ & 1.16 & 1.23 & 0.5 \\
\hline d (fictitious) & 2.20 & 1136 & 0.01 & 270 & $2,460,000$ & $0.0157\left(5 M_{\oplus}\right)$ & $0.178\left(2 R_{\oplus}\right)$ & 0.3 \\
\hline
\end{tabular}

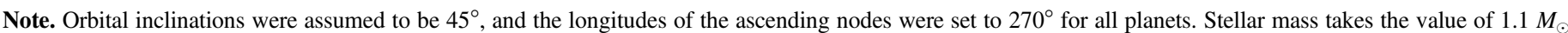

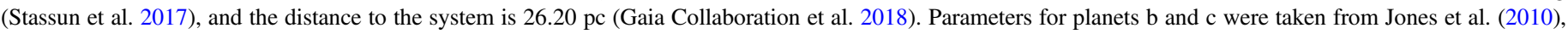

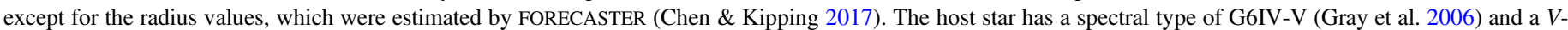
band magnitude of 6.46 (Høg et al. 2000).

summary of the orbital parameters of the fictitious planet are listed in Table 1; its exact location in Figure 1 is plotted as well. We also ran an $N$-body simulation within REBOUND using the exact same parameters in Table 1 to check the time evolution of all orbits and obtained stable results for all planets over a 10 million yr timescale.

\section{Retrieval Tests}

To further verify that the injected fictitious planet within the Region in Figure 1 in the HD 134987 system is indeed below the RV and above the direct imaging detection limits, we conducted retrieval tests for each method. For each one, we predicted the chance of detection of the planet for each method based on detection limit equations and then carried out the retrievals with realistic noise components.

\subsection{Radial Velocity Retrieval}

\subsubsection{Detectability}

The RV semiamplitude $(K)$ of the star's induced oscillation by the planet around it usually is a good indicator of whether the planet could be detected by RV instruments with certain sensitivities that could reach down to a minimum $K$ value. Using the orbital parameters of the fictitious planet in Table 1 and Equation (1), we calculated the semiamplitude induced by this planet to be about $0.203 \mathrm{~m} \mathrm{~s}^{-1}$. This value is below the lowest nominal semiamplitude limit of $0.3 \mathrm{~m} \mathrm{~s}^{-1}$ for current RV instruments that we employ, as indicated in Figure 1, and suggests that the planet would be extremely difficult to retrieve, especially with the addition of noise.

\subsubsection{Retrieval}

The original RV data set published in Jones et al. (2010) contains 138 observations taken by the HIRES and UCLES spectrographs on Keck and the Anglo-Australian Telescope. The data set spans almost 5000 days with instrumental precisions on the order of 1 and $2 \mathrm{~m} \mathrm{~s}^{-1}$, respectively, and no additional signals were found other than the two known planets. To test whether RVs with higher instrumental precision around $0.3 \mathrm{~m} \mathrm{~s}^{-1}$ can recover the signal of our fictitious planet, we created a synthetic RV data set with a time range of 5000 days and a total of 150 data points, similar to the original data set. To mimic the uneven sampling of RV, we increased the number of evenly separated time stamps to $50 \%$ more than the originally intended 150 data points, totaling 225 . These time stamps were then passed through a Gaussian filter with a sigma of 10 days to increase the variation between time stamps. The synthetic RV at each time stamp was then calculated for each planet by solving Kepler's equations using the orbital

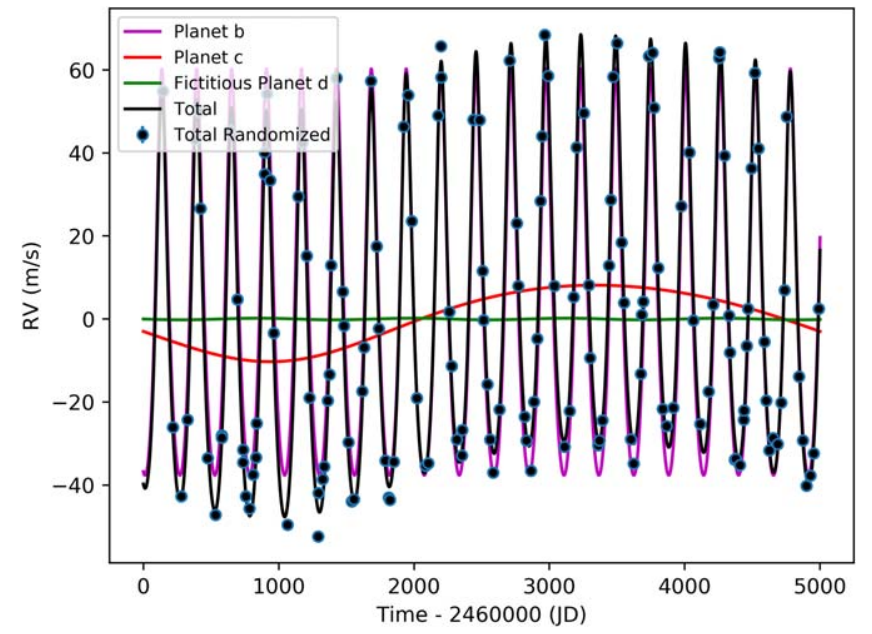

Figure 3. Synthetic RV data set to be used for retrieval. Data points are randomized to include instrumental and astrophysical noise. The black curve shows the total RV variation, and each colored curve represents velocity contributions from the individual planets. Error bars for data points are too small to be seen in the plot.

parameters of all three planets listed in Table 1 and Equation (4), where $V_{0}$ is the velocity offset, which is taken to be zero in our case, and $f$ is the true anomaly of the planet, calculated numerically at each time step for each planet:

$$
V=V_{0}+K[\cos (\omega+f)+e \cos \omega] .
$$

The total RV for the system is simply the superposition of the individual velocity components from each planet. To mimic the RV scatter due to noise, all of the data points were passed through another Gaussian filter, with the sigma variation being the quadrature sum from an instrumental uncertainty of $0.3 \mathrm{~m} \mathrm{~s}^{-1}$ and a host star jitter of $3.5 \mathrm{~m} \mathrm{~s}^{-1}$ (Butler et al. 2006). Finally, the additional $75 \mathrm{RV}$ data points introduced at the beginning were taken out at randomly selected time stamps to further increase the randomness in sampling for the remaining $150 \mathrm{RV}$ points. The final synthetic RV data points created, as well as the velocity components from each planet, are shown in Figure 3.

The RV retrieval of the fictitious planet was carried out by first passing the data set through a periodogram to search for any significant periodic signals. We used the package RVSearch (Rosenthal et al., 2021), an RV periodogram tool that iteratively searches for periodic signals in the time-series $\mathrm{RV}$ data set given a grid of period space. It uses the change in the Bayesian information criterion ( BIC) between the model at the current grid and the best-fit model as a measure of the goodness of the fit. The signal was considered significant if it peaks above the $0.1 \%$ false-alarm probability (FAP) level. 


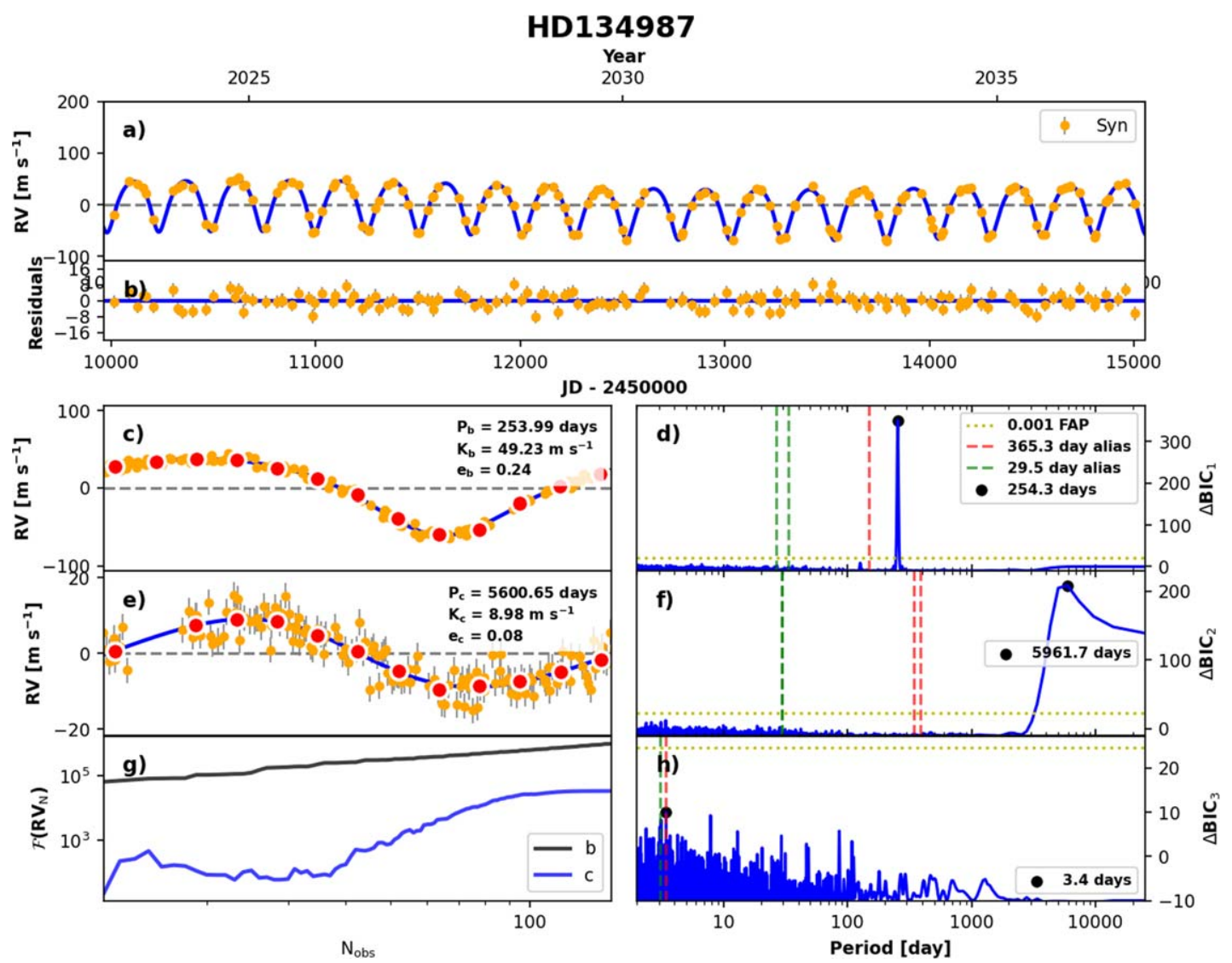

Figure 4. The RVSearch result of the synthetic RV data set. Panels (a) and (b) show the best fit of the data set and the residual. The bottom panels show the phasefolded fit for the planets detected by the search on the left and their corresponding periodogram search results. Two known giant planets are clearly recovered by RVSearch, but there is no apparent signal for the injected fictitious planet.

Figure 4 shows the result of the search. It is apparent that the two known giant planets were successfully recovered by the search, well above the $0.1 \%$ FAP level. However, RVSearch did not find any significant periodic signals in the residual of the two-planet fit, and there are no peaks around the period of the injected fictitious planet.

In addition to the periodogram search, we also used the RV modeling toolkit RadVel (Fulton et al. 2018) to search for the third signal in the synthetic data set. RadVel employs maximum a posteriori optimization for RV fitting and Markov Chain Monte Carlo (MCMC) with robust convergence criteria for confidence interval estimation. For the retrieval, we provided orbital parameter priors for all three planets using values from Table 1 and fitted for the three-planet model. The MCMC was run with eight independent ensembles in parallel with 50 walkers per ensemble, where each walker can take up to 10,000 steps. The run is considered finished if all of the convergence criteria are met or the maximum number of steps for all of the walkers are achieved. The MCMC run on the three-planet model finished with the maximum number of steps taken, and the convergence was not met, indicating that the inclusion of the third planet may not be a good fit to the model. With the results from RVSearch and RadVel, we concluded that the injected fictitious planet that lies below the RV instrumental precision cannot be successfully retrieved.

\subsection{Direct Imaging Retrieval \\ 4.2.1. Detectability}

A planet-star flux ratio can be estimated with a Lambert sphere assumption using Equations (2), (3), and 5 (below), where $\alpha$ is the phase angle of the planet, $\omega$ and $f$ are the argument of periastron and true anomaly of the planet, and $i$ is the inclination of the orbit:

$$
\cos \alpha=\sin (\omega+f) \sin i .
$$

We chose a geometric albedo of 0.5 for both known giant planets $\mathrm{b}$ and $\mathrm{c}$ and 0.3 for the fictitious planet. The chosen albedo values are consistent with previous estimates and models for Jupiter and Neptune analogs (Cahoy et al. 2010). Although planet $\mathrm{b}$ orbits much closer to the host star than a typical cold gas giant and its geometric albedo value is likely to be much lower than 0.5 (Kane \& Gelino 2010), its detection in direct imaging products of either SRP or HabEx is unlikely because its projected orbit lies well outside the starshade FOV for both SRP and HabEx.

We created a code that visualizes the orbits of planetary systems in both top-down and sky projection views, where planetary orbits are color-coded with the planet-star flux ratios that are above the provided instrumental contrast limit as seen from Earth at each orbital position based on Lambert sphere flux ratio calculations (Figure 5). The top-down view also includes a visualization of the conservative and optimistic HZ (CHZ and OHZ, respectively), for which the boundaries are defined in Kopparapu et al. (2013, 2014), and the sky view 

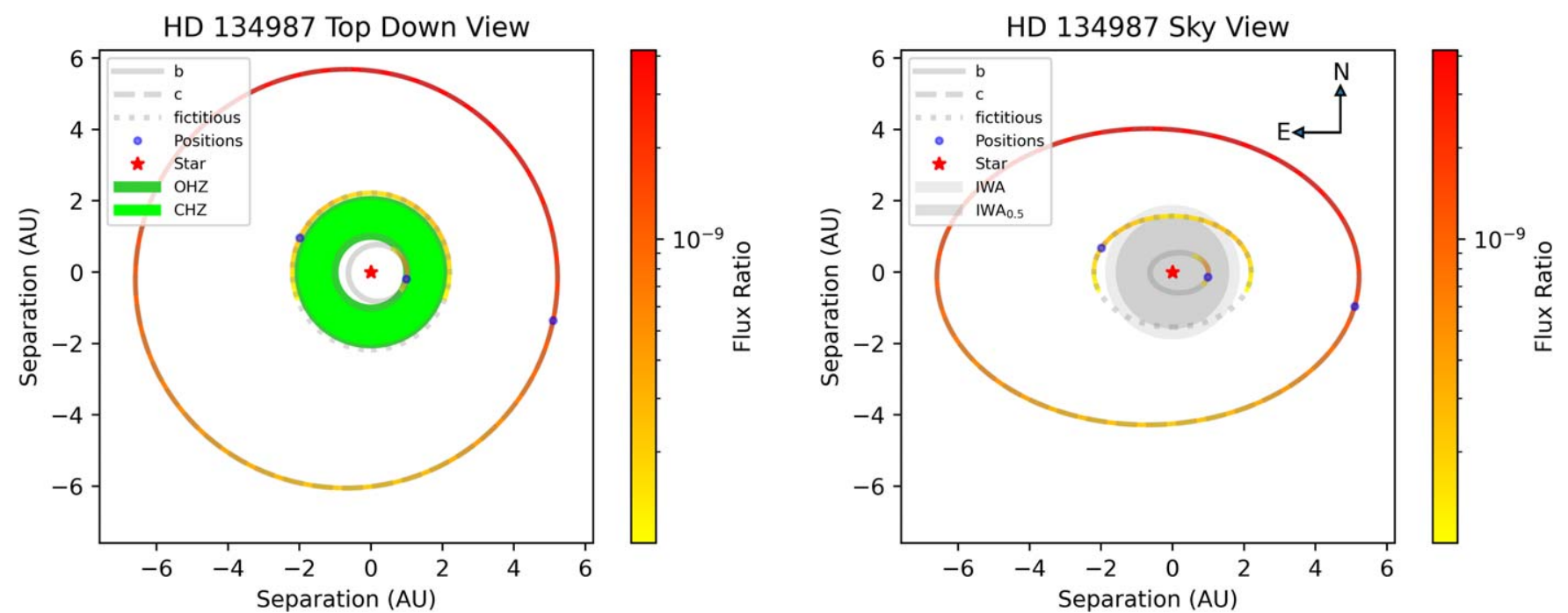

Figure 5. Top-down (left) and sky projection (right) views of the HD 134987 system for Roman's SRP. Planets b and c are known giant planets, and the injected fictitious planet sits in between the two. Orbits are color-coded at locations where the calculated flux ratio values are above the $1 \times 10^{-10}$ flux ratio limit. The sizes of IWA and IWA ${ }_{0.5}$ can be seen in the sky projection view, where the inner $b$ planet is completely covered by IWA $\mathrm{A}_{0.5}$, while the fictitious planet crosses in and out of the IWA. Tiny dots on all three orbits indicate the location of the three planets when retrievals are carried out.

includes the projection of both geometric IWA and the IWA $\mathrm{I}_{0.5}$. The definition of the IWA was given in Section 1 and is simply the angular radius of the starshade as seen from the telescope. On the other hand, the $\mathrm{IWA}_{0.5}$ is defined as the angular radius from the starshade center where the starshade transmittance is $50 \%$; see Figure 6. That is, the ratio between the flux of a pointlike source with the starshade in place or without it is $50 \%$. Given a starshade-telescope distance, the IWA does not depend on the wavelength, whereas the $\mathrm{IWA}_{0.5}$ does, although in a weak fashion. For instance, across the whole visible passband of HabEx (450-975 nm), its value changes by 4\%; see, e.g., Figure 6.4-3 in Gaudi et al. (2020). In the sequel, we adopted the average value of IWA $_{0.5}$ across the passband for both SRP, IWA $0.5=60.7$ mas, and HabEx, IWA $\mathrm{IW}_{0.5}=56.4$ mas. Planets that orbit within the IWA $\mathrm{A}_{0.5}$ and IWA could still be detected. However, below the IWA 0.5 , the optical effects of an imperfect starshade that are not adequately simulated could impact the conclusions, and it is not considered in the literature. Thus, the IWA $\mathrm{IW.5}$ could be considered a reasonable proxy for the minimum separation from the star for exoplanet detection (Gaudi et al. 2020). The code also makes an alternative view of showing the planets' visibility by plotting out an individual planet's flux variation throughout the entire orbital phase, along with the contrast ratio detection threshold as well as IWA and $\mathrm{IWA}_{0.5}$ of the instrument (Figure 7). Occulter transmittance $(\tau)$ can also be incorporated for more accurate flux ratio calculations. If provided with time epochs for observation, planetary positions can be overplotted on all plots indicating the locations of all planets and estimated flux ratios during the time of observation. A detailed summary including time of observation, orbital phase, phase angle, sky projection coordinates, angular separation, and flux ratio of all planets in the system can also be saved in a separate text file.

Using this code along with the orbital parameters in Table 1, we estimated the detectability of our fictitious planet through Roman's SRP and HabEx imaging missions. We left Roman's CGI out of consideration because the detection of the fictitious planet is far beyond the capability of the instrument (Figure 1). Shown in Figure 5 are the orbit and flux ratio plots of the HD

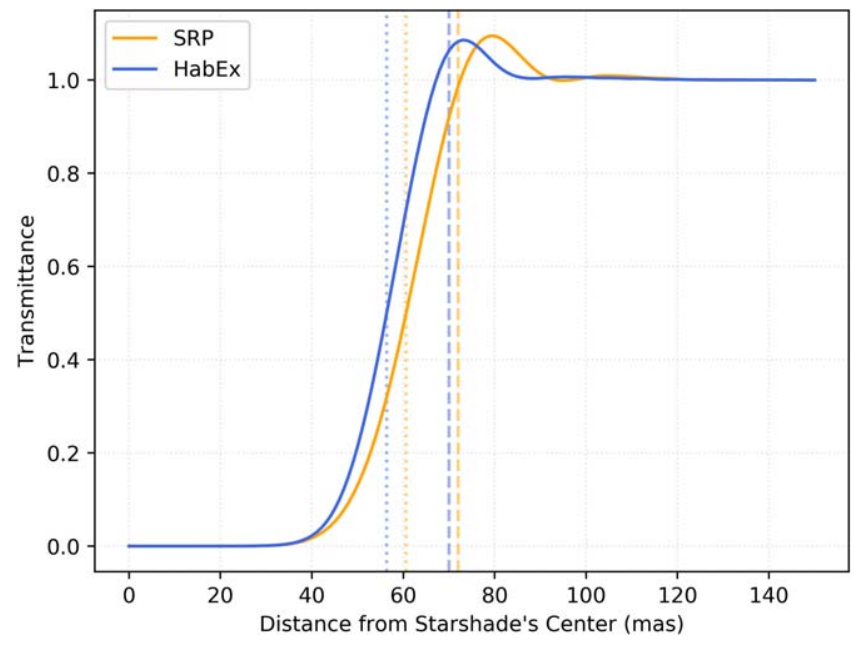

Figure 6. Transmittance profiles of starshades averaged over respective bands for both Roman's SRP and HabEx mission concepts. The vertical dashed and dotted lines are the locations of IWA and IWA $\mathrm{A}_{0.5}$ for both instruments. Values of the transmittance above 1 near the IWA are a known effect of Fresnel diffraction, which is the diffraction regime that applies to the starshades considered in this work.

134987 system with the fictitious planet for Roman's SRP mission concept in both top-down and sky projection view. Similar plots for HabEx are not shown here because the differences in fluxes from SRP are indistinguishable by eye. For the SRP mission, we assumed usage of the blue band $(425-552 \mathrm{~nm})$ for planetary detection with an IWA of 72 mas (Hildebrandt et al. 2021). For HabEx, we used its broad visible channel with a 450-975 nm passband with an IWA of 70 mas. Instead of using the ideal $4.0 \times 10^{-11}$ as the minimum flux ratio limit, we used the more conservative $1.0 \times 10^{-10}$ required minimum detection flux ratio for both SRP and HabEx in anticipation that the imperfection in the manufacturing of the starshade would potentially raise the contrast limit (Seager et al. 2019; Gaudi et al. 2020; Romero-Wolf et al. 2021). For our direct imaging retrieval, we assumed $\mathrm{IWA}_{0.5}$ as the reference limit beyond which one can make a planetary 


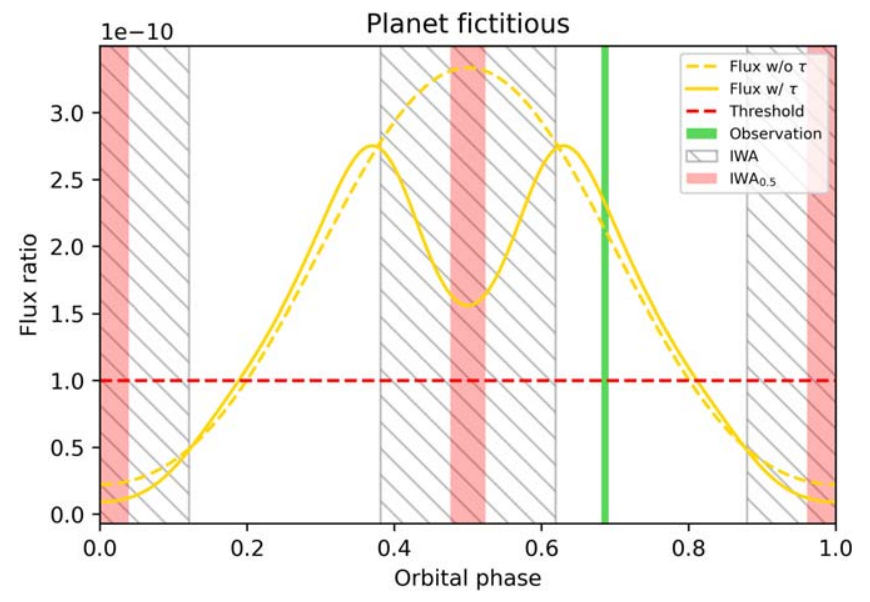

Figure 7. Individual flux variation plot for the fictitious planet in the case of SRP as it makes one complete orbit around the host star. Orbital phase zero here is defined as the passage of periastron. Both fluxes with (solid) and without (dashed) the presence of starshade are displayed to show the difference caused by the starshade's transmittance $(\tau)$. Phases covered by IWA and $\mathrm{IWA}_{0.5}$ are marked with gray hatches and light red bands, respectively. The green band indicates the time/phase when the retrievals are carried out.

detection. To properly account for the flux calculations inside and near the edge of the IWA, we incorporated the transmittance profiles of the starshades for both the Rendezvous (425-552 nm) and HabEx (450-975 nm) cases (Figure 6).

The inner b planet, as expected, is completely covered by the IWA $_{0.5}$ of the instrument and thus not visible, even though for some parts of the orbit, the flux ratio is still very high. The outermost planet is bright and separated enough from the host star that the entire orbit is above the threshold and visible. The fictitious planet orbits at the outer edge of the $\mathrm{OHZ}$ and crosses in and out of the IWA due to orbital inclination (Figure 5). The reflected flux of the fictitious planet varies throughout the orbit, where it is bright enough for the most part of the orbit and falls below the instrumental contrast threshold when near inferior conjunction. The solid yellow curve in the individual flux ratio plot (Figure 7) for the fictitious planet reveals that the planet would cross inside the IWA $\mathrm{IW}_{0.5}$ during its conjunction phases of the orbit. Beyond the $\mathrm{IWA}_{0.5}$, the planet is still bright enough to be detected, despite being inside the IWA. The planet can be seen to achieve its peak apparent brightness at positions slightly beyond the edge of the IWA, reaching an apparent flux ratio value of around $2.8 \times 10^{-10}$ for SRP. For comparison, the planet-to-star flux ratio throughout the entire orbit without the starshade's occultation is shown in Figure 7 as a dashed yellow curve. Flux ratio and planet detectability estimates were also carried out for HabEx but are not shown here due to similar results to SRP, except for configurations of the IWA and IWA $_{0.5}$ and the flux estimates around its edge. The fictitious planet is bright enough for HabEx detection for most of its orbit and again reaches a peak brightness of around $3 \times 10^{-10}$ beyond the edge of the IWA.

Both SRP and HabEx may conduct blind searches of new exoplanet candidates within known systems. The chance of detecting an undiscovered companion through such a search is affected not only by its peak brightness but also by the percentage of an orbit the companion could stay above a certain flux ratio threshold and be visible to the instrument. To determine the direct imaging search completeness for the fictitious planet, we injected the planet at different locations in the orbit and calculated the expected flux ratio values at those
Table 2

Search Completeness of the Fictitious Planet at Different Flux Ratio Thresholds

\begin{tabular}{lcc}
\hline \hline Flux Ratio Threshold $\left(\times 10^{-10}\right)$ & Roman $(\%)$ & HabEx $(\%)$ \\
\hline 1.0 & 56 & 61 \\
1.25 & 50 & 55 \\
1.5 & 45 & 50 \\
1.75 & 39 & 45 \\
2.0 & 31 & 40 \\
2.25 & 24 & 36 \\
2.5 & 16 & 25 \\
2.75 & 2 & 17 \\
3.0 & 0 & 7 \\
\hline
\end{tabular}

Note. Completeness is indicated by the percentage of the planet's orbit that has flux ratios above the different thresholds being considered. Calculations are for the Roman SRP and HabEx starshade observations. Apparent flux ratios lower than $1.0 \times 10^{-10}$ are not included due to the too-long integration time needed. The peak apparent flux ratio of the fictitious planet can reach $2.75 \times 10^{-10}$ for SRP and $3.07 \times 10^{-10}$ for HabEx.

locations. The planet is considered detected if its flux ratio at the injected location is higher than certain flux ratio thresholds. We computed this for nine flux ratio thresholds from $1.0 \times 10^{-10}$ to $3.0 \times 10^{-10}$. Table 2 lists the percentage of the fictitious planet's orbit where its flux ratio is higher than the different thresholds considered for both SRP and HabEx starshade configurations. A higher percentage indicates that the fictitious planet would be more likely to be detected through an imaging search for the system. Although flux ratios below $1.0 \times 10^{-10}$ could be detected by both instruments in optimistic scenarios, the required integration time becomes closer to the available observing window due to solar avoidance angles for SRP (Seager et al. 2019; Romero-Wolf et al. 2021). As can be seen in Figures 5 and 7 and Table 2, the fictitious planet is clearly bright enough to be above the nominal instrumental detection thresholds for both SRP and HabEx. As expected, the fictitious planet at its brightest times only covers a tiny fraction of its orbit, making detection at its peak brightness unlikely. However, the chance of detection increases as the flux ratio threshold decreases, for which at least half of the planet's orbit can be detected when the planet has a flux ratio of at least $1.25 \times 10^{-10}$ for Roman and $1.5 \times 10^{-10}$ for HabEx. It is worth noting that at each flux ratio threshold, the planet consistently has a larger chance of detection for HabEx observations than for SRP, thanks to its slightly smaller IWA and different transmittance profile than SRP's (Figure 6).

\subsubsection{Retrieval}

A more realistic detection estimation should take into account different noise sources both astrophysical and instrumental, such as background stars and sky noise, residual starlight, detector noise, clock-induced charge, quantum efficiency, optical throughput, etc. For direct imaging retrieval of the fictitious planet, we carried out simulations using the SISTER (Hildebrandt et al. 2021) package to create and retrieve the planet's signal with both SRP and HabEx configurations. SISTER is a versatile toolkit that provides accurate models of exoplanet images when observed with a starshade. The tool allows inputs of various observational parameters, including both astrophysical and instrumental, for realistic starshade simulations. It has been used to generate the 
SRP and HabEx simulations of the Starshade Exoplanet Data Challenge (Hu et al. 2021).

For the SISTER simulations, we selected the times when the fictitious planet has apparent flux ratios around $2.25 \times 10^{-10}$. At this brightness, the integration times of both instruments are well below the maximum observing times while ensuring there is still a good chance of detecting the fictitious planet through a blind search within the system. According to Table 2, the planet would have a brightness above this level for about a quarter of its orbit. We used this same flux ratio for both SRP and HabEx for direct side-by-side comparison of the imaging capabilities of both instruments. We further calculated the positions of the fictitious planet when this flux ratio would occur to make sure the planet is not within the $\mathrm{IWA}_{0.5}$. The positions can be seen as blue dots in Figure 5 and the vertical green band in Figure 7.

The simulations assumed instrumental parameters consistent with the current best estimates of the starshade nonideal performance (Seager et al. 2019; Gaudi et al. 2020; Hildebrandt et al. 2021; Romero-Wolf et al. 2021). We included imperfect starshade petal edges from manufacturing and deployment errors consistent with mission requirements leaving a residual starlight equivalent to a $1.4 \times 10^{-10}$ contrast ratio, a few times higher than the ideal limit of SRP and HabEx. Solar glint (McKeithen et al. 2021) was computed under the assumption that the starshade edges are coated with an antireflection coating similar to one that has been tested in the laboratory. The Sun is $60^{\circ}$ from the starshade normal, which is a median value among planned observations, and is oriented at $45^{\circ}$ from horizontal. We included a pointing jitter of the telescopes (14 mas for SRP and 2 mas for HabEx) consistent with the expected in-flight performance of the attitude control systems. Regarding the optical performance of SRP and HabEx, we incorporated the optical throughput and expected losses as described in the corresponding reports. The performance of the Roman detectors was assumed to be in its end-of-life condition, since the SRP is not expected to be paired up with Roman until near the end of the telescope mission. HabEx instrumental conditions were assumed to be beginning-of-life. In both cases, we assumed an EMCCD detector with an instrumental setup and single exposure consistent with each mission's specifications, including quantum efficiency, dark current, clockinduced charge noise, readout noise, and other loss effects. The simulations also included local zodiacal light (with a surface brightness of $23 \mathrm{~V}$ mag $\operatorname{arcsec}^{-2}$ ), as well as a smooth exozodiacal cloud resulting from a simulation performed with ZODIPIC $^{10}$ a zodiacal cloud simulation tool that incorporates observational data from the solar system. The dust density was chosen to be five times that of the solar system, and we chose a similar asymmetry factor as in the solar system to simulate the forward scattering effect with $g=0.2$, where $g$ is the asymmetry factor for the Henyey-Greenstein phase function; see, e.g., Section 3.2 in Stark (2011). For the background object, we did not add any galactic background stars, but we selected the brightest background galaxy found in the Haystack data cubes (Roberge et al. 2017). The resulting starshade images of both SRP and HabEx with all components are shown in the top panels of Figure 8.

\footnotetext{
${ }^{10}$ The ZODIPIC package can be obtained at http://ascl.net/1202.002. We used a version of ZODIPIC with a more accurate handling of the forward scattering term; see Roberge et al. (2017).
}

The total integration time was chosen to provide a signal-tonoise ratio $(\mathrm{S} / \mathrm{N})$ of 5 for both SRP and HabEx to show what a detection would look like, shown in the middle panels of Figure 8, as well as the bottom panels, where the images are zoomed in around the location of the fictitious planet. The total integration time for SRP is of the order of 7 days, whereas for HabEx, it is of the order of $6 \mathrm{hr}$. The main factors that reduce the integration time between HabEx and SRP are, by relevance, (i) the optical collecting area in HabEx, which is about 3.5 times larger than that in SRP; (ii) the broader bandwidth in HabEx's visible channel (450-975 nm) compared to SRP's 425-552 nm channel, although the actual value depends on the star's spectrum and planet's albedo; (iii) the optical efficiency of the system, where HabEx approximately doubles that of SRP; (iv) better detector properties (quantum efficiency and noise properties); and (v) a better disambiguation of the signal of the exoplanet from that of diffuse background sources in the case of HabEx - in particular, exozodiacal light-due to HabEx's finer angular resolution. Summarizing all of these factors in the case of the fictitious planet, the photon detection rate in HabEx's visible channel is about nine times that of SRP's 425-552 nm channel. The calculation of the $\mathrm{S} / \mathrm{N}$ was performed using aperture photometry under the assumption that an unbiased yet noisy estimation of all nonplanetary signal can be subtracted from the noisy simulation with all components present. These nonplanetary components are, by relevance, the exozodiacal cloud, the local zodiacal light, the solar glint (McKeithen et al. 2021), and residual scattered starlight from the imperfect starshade. For instance, when the fictitious planet is near the IWA with a planet-star flux ratio of $\sim 2.5 \times 10^{-10}$ (see Figure 7), the relative weight of each component compared to the total signal in a box with a side length of 1 FWHM of the average PSF response is, approximately, exozodiacal cloud ( $\sim 60 \%)$, local zodiacal light $(\sim 15 \%)$, solar glint and residual starlight from the imperfect starshade $(\sim 18 \%$ in the case of SRP and $\sim 9 \%$ in the case of HabEx), and planetary signal ( $\sim 7 \%$ in the case of SRP and $\sim 16 \%$ in the case of HabEx). Notice that the spatial morphology of each component is different. In particular, the brightness of the local zodiacal light is the same across the image given the small angular size of the simulation, except for intrinsic fluctuations, i.e., its shot noise. The imaging simulation software SISTER (Hildebrandt et al. 2021) takes care of each of them adequately. In practice, this is equivalent to a simulation with the planetary signal and its shot noise component, together with the shot noise contribution from the nonplanetary signal and the detector noise, both increased by a factor of $\sim \sqrt{2}$ because all noise sources are assumed to follow either a Poisson or Gaussian probability distribution (Figure 8). This is consistent with the methodology in SRP and HabEx reports, as well as other similar investigations (Robinson et al. 2016; Feng et al. 2018; Seager et al. 2019; Damiano et al. 2020; Gaudi et al. 2020; RomeroWolf et al. 2021). Our estimates are consistent with the ones derived in such publications. Since the reference flux ratio value for the fictitious planet is greater than or equal to $2.25 \times 10^{-10}$, which is higher than the residual starlight fluctuations due to the imperfect starshade, $\sim 1.4 \times 10^{-10}$, the $\mathrm{S} / \mathrm{N}$ could be increased at the expense of increasing the integration times. Clearly, that is not an issue for HabEx. 

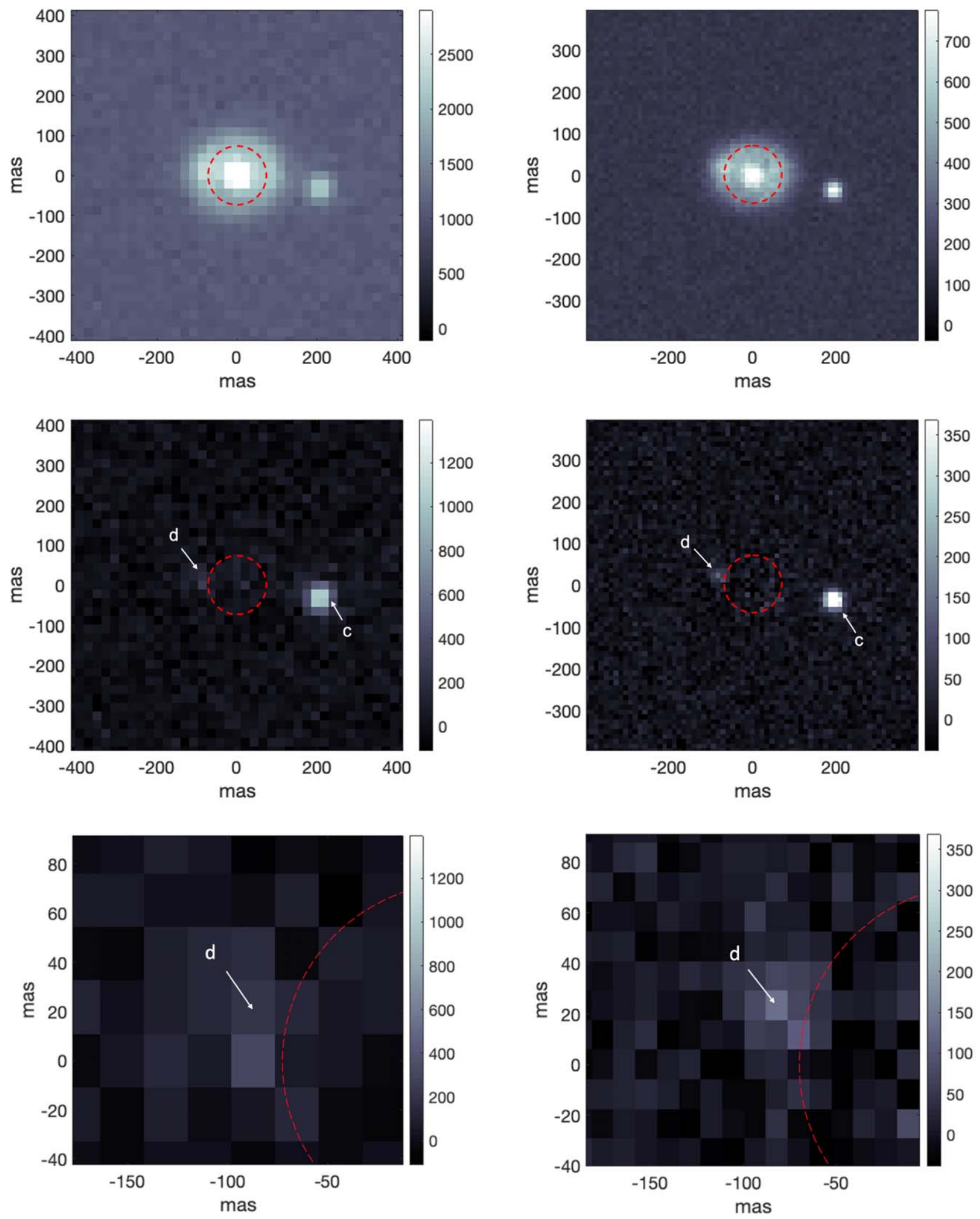

Figure 8. The panels in the left column show the SISTER simulation for SRP of the planetary scenario around HD 134987 with detector pixel scales of 21.8 mas $\mathrm{pixel}^{-1}$. The panels in the right column show similar simulations for HabEx with pixel scales of 11.7 mas pixel ${ }^{-1}$. The integration time was chosen to recover an $\mathrm{S} / \mathrm{N}$ $=5$ for the fictitious planet. The top panels are simulations with all of the components, both astrophysical and instrumental. The middle panels show only the planetary signals after the subtraction of unbiased nonplanetary signals; see text. The bottom panels show the zoomed-in view around the location of planet d, which is the fictitious planet we injected into the system. The color bars representing the level of brightness are in units of integrated photoelectrons. Arrowheads in the bottom panels point to the exact position of the fictitious planet in those images. Our fictitious planet is not clearly visible before the noise subtraction in the top panels, but it is revealed afterward. Planet $\mathrm{b}$ is not visible in all cases, since it is inside the IWA 0.5 of the instrument. Planet $\mathrm{c}$ is clearly visible in all situations. 


\section{Discussion}

Although SRP and HabEx both have similar required contrast ratio limits of $1 \times 10^{-10}$, the imaging simulation results show that HabEx is clearly superior. At similar flux ratio levels, the integration time required to successfully retrieve the signal of the fictitious planet with an $\mathrm{S} / \mathrm{N}$ of 5 is much shorter for HabEx. As explained in Section 4.2.2, HabEx's larger, unobstructed telescope; broader passband in the visible channel; and other instrumental advantages compared to SRP result in a higher integrated flux of about nine times that of SRP in the case of the fictitious planet around HD 134987. With an integration time of around 7 days for SRP, imaging of our fictitious sub-Neptune planet proves to be a challenging task at a distance of 26.2 pc. A smaller terrestrial planet at a similar distance and separation from the host star would result in even lower planet-star flux ratios, making the required integration time for planetary detection much longer, possibly on the order of 30 days or more, which is not feasible for direct imaging observations. Therefore, imaging an Earth-like counterpart at this distance with SRP is clearly unfavorable, and the detection and characterization of small terrestrial planets are likely to be limited to within $20 \mathrm{pc}$ or closer until the launch of HabEx.

Several assumptions were made, and some of them may affect the result of this work. We discuss the implications of changing those assumptions as follows.

1. Since the inclinations of the two known planets in the HD 134987 system are unknown, here we assumed an inclination of $45^{\circ}$ for all three planets, including our fictitious planet. Varying this value would have consequences for the flux ratios on all planets. If the true inclination of the system is less than $45^{\circ}$, making the orbits more face-on, the fictitious planet would have a larger fraction of its orbit bright enough for detection, even though the peak brightness would not be as bright as the $45^{\circ}$ case. However, too small of an inclination angle would likely eliminate the likelihood of the presence of such a planet in this system, since larger planetary masses boosted by the $\sin i$ factor would diminish the already narrow dynamically allowed parameter space (Figure 2). On the other hand, if the true inclination is more toward edge-on, more parts of the orbit would be within the IWA or $\mathrm{IWA}_{0.5}$, costing the chance of detection in this case, which is not ideal for direct imaging. In addition, if a different inclination value was selected for the study, or the true inclination of the system obtained through other methods in the future indicates a drastically different angle, the size of the Region in Figure 1 would vary accordingly, as would the allowed parameter space for the fictitious planet. The RV curves would shift further toward smaller or larger separation for more face-on or edge-on orbits, respectively. In the case of HD 134987 , the Region exists in all cases, and our fictitious planet is valid for the purpose of this study, which is to probe the parameter space in between RV and imaging sensitivities, no matter what inclination was picked. However, this might not be true if similar studies were to be carried out for other systems, and a more careful selection of the inclination angle might be needed in other cases.

2. The physical and orbital parameters of the fictitious planet make an impact on its detectability for direct imaging. Here we assumed a near-zero eccentricity for our planet for convenience. However, as pointed out in Kane (2013), at the same separation for the star, higheccentricity planets could improve its chance of detection. In addition, the fictitious planet could take on different radius values and thus different mass values. The allowed radius range for the purpose of this work according to Figure 1 is roughly between 1 and $2.5 R_{\oplus}$, for which the masses are predicted to be between 1 and $7 M_{\oplus}$. A higher radius for the planet would have increased the reflected flux, making them easier to detect, but the location of the planet has to be farther away from the star in order to stay in between the RV and direct imaging detection thresholds (Figure 1) that we are interested in. The result of the two might not necessarily make the fictitious planet any more favorable for detection than the current configuration. When working on known planets that only have mass information, either in the HD 134987 or other systems, the deviation from the true value using any mass-radius relationship may have an impact on the detectability if such a planet lies near the detection threshold of any imaging instruments.

3. The simulation of a realistic geometric albedo of the two known planets and the fictitious planet is beyond the scope of this paper. Instead, we assumed a geometric albedo of 0.5 for the two known giant planets and 0.3 for the fictitious planet. This assumption is based on previous works, such as Cahoy et al. (2010) and Kane \& Gelino (2010), estimating the albedo spectra of solar system analogs and albedo variation of giant planets with respect to orbital distances. A more careful albedo treatment can be obtained through atmospheric modeling for each planet. Higher geometric albedo values for planets would result in a higher chance of detection.

It is worth noting that this work is by no means dictating the presence of additional yet to be discovered planets in the HD 134987 system. Rather, it shows that such a discovery is entirely possible with future direct imaging missions thanks to the complementary detection sensitivity of direct imaging to RVs. Although the work presented here could only be applied to one system at a time, in this case the HD 134987 system, a similar procedure could be carried out for other direct imaging systems with different parameters and assumptions. This indepth look at a particular system with the combination of different detection sensitivity curves, along with realistic RV, imaging, and dynamical simulations, allows the parameter space within the system to be fully explored and provides unique system information that other statistical studies cannot offer. This is particularly the case when more than one known planet exists within the system, where the known bodies can provide constraints on the physical and orbital properties of the possible planetary candidate in between the orbits of known planets through dynamical simulations or completely rule out such a likelihood, therefore saving telescope time from searching for new exoplanet candidates from ruled-out systems. Results from similar studies for other direct imaging systems, if carried out, could be valuable tools for determining systems with a likelihood of hosting additional planets, predicting the best time and instruments to recover such potential planets, informing the target selection process of future imaging missions, and maximizing the limited resources of starshade operation in the hunt for new planets. 


\section{Conclusion}

In this work, we demonstrated the steps in trying to predict whether new planets that are below the RV detection thresholds can be discovered by future direct imaging missions in one particular case of the HD 134987 system, one of the farthest systems to be imaged by future missions. We used the HD 134987 system as a test case and developed a detection threshold figure that combines sensitivity curves from both RV and direct imaging. This figure (Figure 1) was constructed with the most optimistic scenario and does not take into account the dynamical influence of known bodies in the system, as well as additional noise sources such as stellar jitter, starshade imperfection, and local and exozodiacal light. Thus, a careful examination of the system is necessary to verify the detection limit. We have considered some detailed simulations that take into account system stability, as well as realistic starshade images with an imperfect starshade within the mission requirements. Based on Figure 1, we were able to locate the parameter space to search for the location of the potential new planet through dynamical analysis. We assigned a sub-Neptune fictitious planet and injected it into the HD 134987 system for retrieval tests. An RV periodogram search and modeling on a synthetic RV data set returned negative results, as expected, while direct imaging retrieval was able to successfully recover the signal of the fictitious planet. Through imaging simulations, we were able to directly compare the imaging capabilities of SRP and HabEx, and the results show that imaging with HabEx is clearly superior and that SRP is probably not capable of imaging low-mass planets at a distance of around 26 pc. Since the majority of the future imaging candidates are RV planets, the synergy between RV and imaging becomes ever-increasingly crucial. These predicted directly imaged planets would provide potential discoveries of cold giant planets, subgiant planets at long orbital periods, and even terrestrial planets in the $\mathrm{HZ}$, increasing the science return of the missions, as well as opportunities for atmospheric characterization in the low incident flux regime.

The authors would like to thank Chris Stark for sharing an improved version of ZODIPIC, which was used to generate the input exozodiacal dust in Section 4.2.2. The authors wish to also thank the referee for providing a swift and valuable response that greatly improved the presentation of this work. This work was funded by the Roman CGI Science Investigation Team contract \#NNG16P27C (PI: Margaret Turnbull). This research has made use of the NASA Exoplanet Archive, which is operated by the California Institute of Technology, under contract with the National Aeronautics and Space Administration under the Exoplanet Exploration Program. Dynamical simulations in this paper made use of the REBOUND code, which is freely available at http://github. com/hannorein/rebound.

Software: RadVel (Fulton et al. 2018), RVSearch (Rosenthal et al. 2021), REBOUND (Rein \& Liu 2012), SISTER sister. caltech.edu (Hildebrandt et al. 2021).

\section{ORCID iDs}

Zhexing Li (i) https://orcid.org/0000-0002-4860-7667 Sergi R. Hildebrandt (D) https://orcid.org/0000-00030220-0009

Stephen R. Kane (iD https://orcid.org/0000-0002-7084-0529
Neil T. Zimmerman (ib https://orcid.org/0000-0001-5484-1516

Julien H. Girard (1) https://orcid.org/0000-0001-8627-0404 Junellie Gonzalez-Quiles (iD https://orcid.org/0000-00029032-8530

Margaret C. Turnbull (1) https://orcid.org/0000-00020569-1643

\section{References}

Batalha, N. E., Lewis, T., Fortney, J. J., et al. 2019, ApJL, 885, L25 Batalha, N. M., Rowe, J. F., Bryson, S. T., et al. 2013, ApJS, 204, 24 Borucki, W. J., Koch, D., Basri, G., et al. 2010, Sci, 327, 977 Borucki, W. J., Koch, D. G., Basri, G., et al. 2011a, ApJ, 728, 117 Borucki, W. J., Koch, D. G., Basri, G., et al. 2011b, ApJ, 736, 19 Burke, C. J., Bryson, S. T., Mullally, F., et al. 2014, ApJS, 210, 19 Butler, R. P., \& Marcy, G. W. 1996, ApJL, 464, L153

Butler, R. P., Wright, J. T., Marcy, G. W., et al. 2006, ApJ, 646, 505 Cahoy, K. L., Marley, M. S., \& Fortney, J. J. 2010, ApJ, 724, 189 Chauvin, G., Lagrange, A. M., Dumas, C., et al. 2004, A\&A, 425, L29 Chen, J., \& Kipping, D. 2017, ApJ, 834, 17

Cincotta, P. M., \& Simó, C. 2000, A\&AS, 147, 205

Coughlin, J. L., Mullally, F., Thompson, S. E., et al. 2016, ApJS, 224, 12 Damiano, M., Hu, R., \& Hildebrandt, S. R. 2020, AJ, 160, 206

Duncan, M. J., Levison, H. F., \& Lee, M. H. 1998, AJ, 116, 2067

Feng, Y. K., Robinson, T. D., Fortney, J. J., et al. 2018, AJ, 155, 200

Fischer, D. A., Anglada-Escude, G., Arriagada, P., et al. 2016, PASP, 128, 066001

Fulton, B. J., Petigura, E. A., Blunt, S., \& Sinukoff, E. 2018, PASP, 130, 044504

Gaia Collaboration, Brown, A. G. A., Vallenari, A., et al. 2018, A\&A, 616, A1

Gaudi, B. S., Seager, S., Mennesson, B., et al. 2020, arXiv:2001.06683

Gray, R. O., Corbally, C. J., Garrison, R. F., et al. 2006, AJ, 132, 161

Guillot, T. 2005, AREPS, 33, 493

Harness, A., Shaklan, S., Willems, P., et al. 2021, JATIS, 7, 021207

Hildebrandt, S. R., Shaklan, S., Cady, E., \& Turnbull, M. 2021, JATIS, in press

Hinse, T. C., Christou, A. A., Alvarellos, J. L. A., \& Goździewski, K. 2010, MNRAS, 404, 837

Høg, E., Fabricius, C., Makarov, V. V., et al. 2000, A\&A, 355, L27

Howard, A. W., Marcy, G. W., Bryson, S. T., et al. 2012, ApJS, 201, 15

Hu, R., Sergi, R. H., Damiano, M., et al. 2021, JATIS, 7, 021216

Janson, M., Bergfors, C., Goto, M., Brandner, W., \& Lafrenière, D. 2010, ApJL, 710, L35

Jones, H. R. A., Butler, R. P., Tinney, C. G., et al. 2010, MNRAS, 403, 1703

Kane, S. R. 2013, ApJ, 766, 10

Kane, S. R., \& Gelino, D. M. 2010, ApJ, 724, 818

Kane, S. R., Hill, M. L., Kasting, J. F., et al. 2016, ApJ, 830, 1

Konopacky, Q. M., Barman, T. S., Macintosh, B. A., \& Marois, C. 2013, Sci, 339, 1398

Kopparapu, R. K., Ramirez, R., Kasting, J. F., et al. 2013, ApJ, 765, 131

Kopparapu, R. K., Ramirez, R. M., SchottelKotte, J., et al. 2014, ApJL, 787, L29

Lagrange, A. M., Gratadour, D., Chauvin, G., et al. 2009, A\&A, 493, L21

Latham, D. W., Mazeh, T., Stefanik, R. P., Mayor, M., \& Burki, G. 1989 Natur, 339, 38

Luhn, J. K., Wright, J. T., Howard, A. W., \& Isaacson, H. 2020, AJ, 159, 235

Marois, C., Macintosh, B., Barman, T., et al. 2008, Sci, 322, 1348

Mayor, M., \& Queloz, D. 1995, Natur, 378, 355

McKeithen, D., Shaklan, S., Sheikh, D., Balasubramanian, K., \& Lowe, E. 2021, JATIS, 7, 021208

Meunier, N., \& Lagrange, A. M. 2019, A\&A, 625, L6

Meunier, N., \& Lagrange, A. M. 2020, A\&A, 642, A157

Mullally, F., Coughlin, J. L., Thompson, S. E., et al. 2015, ApJS, 217, 31

Pepe, F., Cristiani, S., Rebolo, R., et al. 2021, A\&A, 645, A96

Petigura, E. A., Marcy, G. W., \& Howard, A. W. 2013, ApJ, 770, 69

Rein, H., \& Liu, S. F. 2012, A\&A, 537, A128

Rein, H., \& Tamayo, D. 2015, MNRAS, 452, 376

Ricker, G. R., Winn, J. N., Vanderspek, R., et al. 2015, JATIS, 1, 014003

Roberge, A., Rizzo, M. J., Lincowski, A. P., et al. 2017, PASP, 129, 124401

Robinson, T. D., Stapelfeldt, K. R., \& Marley, M. S. 2016, PASP, 128, 025003

Romero-Wolf, A., Bryden, G., Seager, S., et al. 2021, JATIS, 7, 021210

Rosenthal, L. J., Fulton, B. J., Hirsch, L. A., et al. 2021, AAS, in press

Rowe, J. F., Coughlin, J. L., Antoci, V., et al. 2015, ApJS, 217, 16

Schwab, C., Rakich, A., Gong, Q., et al. 2016, Proc. SPIE, 9908, 99087H

Seager, S., Kasdin, N. J., Booth, J., et al. 2019, BAAS, 51, 106

Spergel, D., Gehrels, N., Baltay, C., et al. 2015, arXiv:1503.03757 
Stark, C. C. 2011, AJ, 142, 123

Stassun, K. G., Collins, K. A., \& Gaudi, B. S. 2017, AJ, 153, 136

Thompson, S. E., Coughlin, J. L., Hoffman, K., et al. 2018, ApJS, 235, 38
Trauger, J., Moody, D., Krist, J., \& Gordon, B. 2016, JATIS, 2, 011013

Vogt, S. S., Allen, S. L., Bigelow, B. C., et al. 1994, Proc. SPIE, 2198, 362 